\title{
Low cost Front end Diplexer for MoCA 2.0 \& DVB-C/C2 Systems
}

\author{
Madhusudana Rao Kothari ${ }^{1}$, Sree Lakshmi Ele ${ }^{2}$ \\ ${ }^{I}$ (Tata power SED, Bangalore and Communications Systems Engineering, Manipal University, India) \\ ${ }^{2}$ (VLSI Design and Embedded Systems Engineering, VTU Extension Centre - UTL Technologies Ltd, India)
}

\begin{abstract}
The Media over Cable Alliance (MoCA) and Digital Video Broad Casting-Cable (DVB-C) are the two different coax based home network standards. At Present, the home networks rely on both the standards, based on the available devices. The Commercial devices for these MoCA2.0 and DVB-C/C2 can be from the different vendors ranging at very high costs. In this study, an attempt has been made to design a low cost Diplexer, which can be tuned further with the commercial available Inductors and capacitors. This helps us to control the cost and size with the designed Diplexer followed by the effective design of home network.
\end{abstract}

Keywords: MoCA 2.0, DVB-C, Diplexer, Home Network.

\section{Introduction}

The Coax based Home Network [1] consists of DVB-C and MoCA devices [8].

\section{A. Media over Cable Alliance (MoCA 2.0):}

The Media over cable Alliance 2.0 is the latest version of the Multimedia over Coax Alliance's powerful standard for transporting data over the existing coaxial wiring in consumer's homes. MoCA is proved to be valuable and capable at present in both the service provider deployments and in consumer networking [5]. MoCA 2.-0 enriches the cable network capacity, flexibility, and better power management network [5]. The MoCA 1.0 specification approved in 2006 demonstrated the untapped potential of residential home coaxial wiring for high performance and proved to be inexpensive in home two-way networking. This technology is very effective because of its bi-directional connectivity to any coax outlet in a house, which is capable of receiving cable or satellite programming, even though the coax wiring intended to deliver the signals in one-way [4]. It can deliver the signals more than $100 \mathrm{Mbps}$ at an aggregate throughput with both the reliability (low packet error rate) and the low latency, needed for high quality multimedia traffic and to separate the other frequencies used by legacy cable and the satellite services on the same coax wiring. MoCA 1.1 has provided some important enhancements to MoCA 1.0 by both the throughput and management. But the most important step in the performance of MoCA has come with MoCA 2.0, with an increase in the aggregate throughput as 1 Gbps (in the channel bonded, point-to-point "turbo" mode) along with enhancements such as new power-saving modes [5].

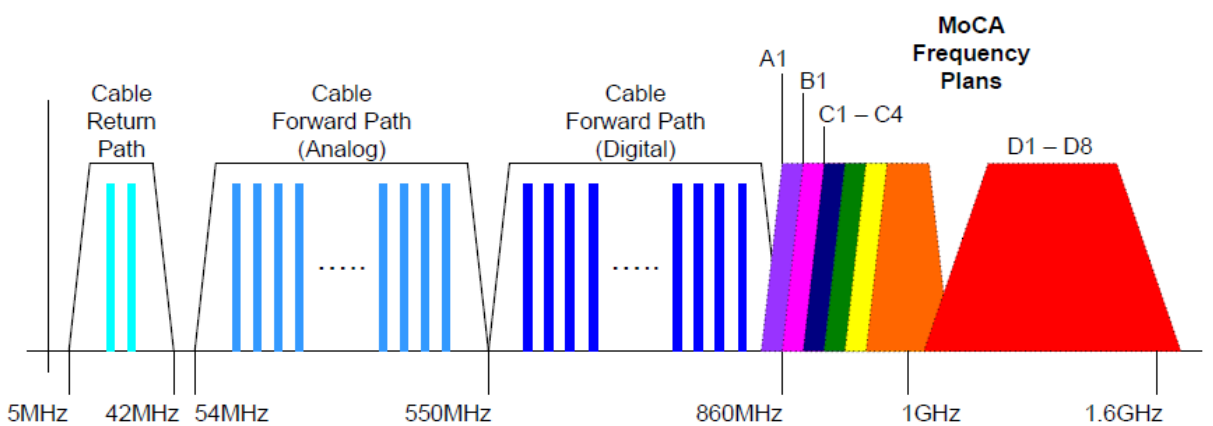

Fig. 1 MoCA frequency Plan (source: MoCA Home Network Installation and Maintenance)

MoCA 2.0 Operates for $50 \mathrm{MHz}$ channels, with a frequency of $850 \mathrm{MHz}$ to $1.675 \mathrm{GHz}$ as shown in the figure1. This has an aggregate Data with Throughput as $400 \mathrm{Mbps}$ while using the single channel and $800 \mathrm{Mbps}$ while using the two bonded channels in Enhanced mode. In Turbo mode, the throughput will be $500 \mathrm{Mbps}$ while using the single channel and $1 \mathrm{Gbps}$ while using the two bonded channels [5]. As shown in figure 2, the MoCA physical Topology tree uses the coaxial cable, passive filters and splitters. The equipment connected to the MoCA network is also known as "nodes of the network" and this is designed to support max $300 \mathrm{ft} / 25 \mathrm{~dB}$ loss (@ $750 \mathrm{MHz}$ ) between root and nodes [5]. 


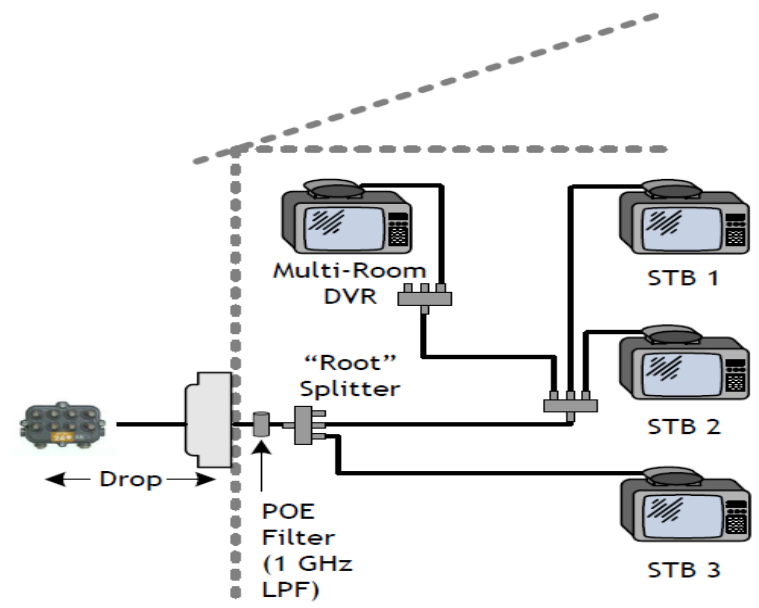

Fig. 2 MoCA Physical Topology (source: MoCA Home Network Installation and Maintenance)

\section{B. Digital Video Broadcasting- Cable:}

DVB-C [6] is introduced by ETSI in December 1994. This subsequently becomes the most widely used transmission system for digital cable television [2]. The standard is deployed worldwide in systems ranging from the smaller satellite master antenna TV (SMATV) systems to larger cable television networks (CATV). DVB-C is also integrated as the physical layer for the European version of DOCSIS (Data over Cable Service Interface Specification). As with its predecessor, DVB-C2 offers a wide range of modes and options that can be optimized for different network characteristics and the requirements of different services planned for delivery to cable customers [2]. By using the state of the art code and modulation techniques, it offers higher spectrum efficiency likely greater than $30 \%$, with the same conditions as today's DVB-C deployments.

The noise performance of DVB-C2 is excellent. Coming close to the Shannon limit, the theoretical maximum information transfers the rate in channel for a given noise level. The figure 3 shows the range and fine granularity of possible solutions. The chosen COFDM modulation scheme is insensitive to echoes caused by typical in-house coaxial networks and very robust in relation to the impulsive noise interference [7]. Notches, narrowband and broadband can be used to cope with different interference scenarios. Flexibility in terms of bandwidth is a further necessary feature of DVB-C2. In the near future, the cable networks deployed with DVB$\mathrm{C} 2$ will allow a wide range of broad signals (e.g. $32 \mathrm{MHz}$ and more) to be transmitted and can meet the operator's requirements for larger pipes, which allows a efficient sharing of the available resources between the individual customers and services.

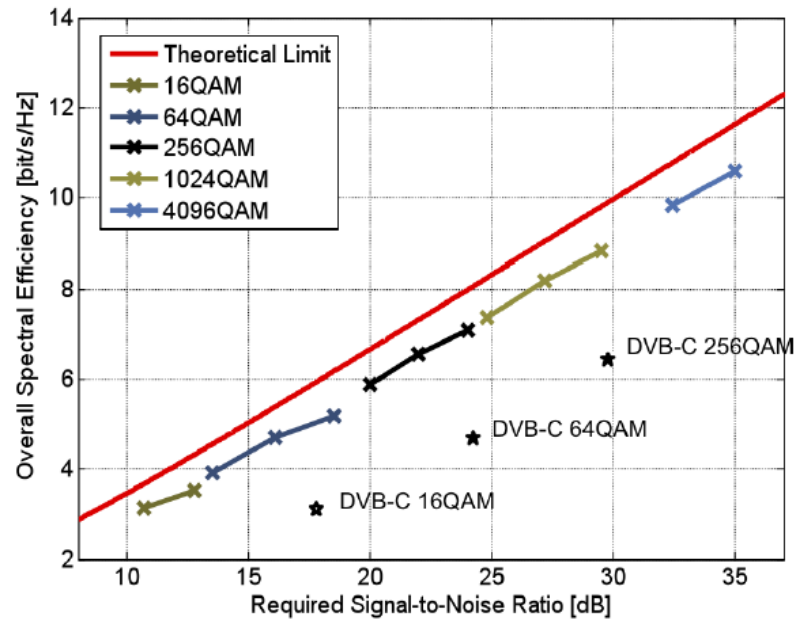

Fig. 3 SNR Graph for different Modulations used in DVB-C/C2 (source: [2] DVB Fact Sheet - July 2012)

\section{Specifications Of Diplexer}

The RF input and output specifications [3] of Diplexer is as follows

1) Return loss at the input port

a. $\quad<870 \mathrm{MHz}: \geq 8 \mathrm{~dB}$

b. Between 975 to $1675 \mathrm{MHz}: \geq 5 \mathrm{~dB}$ 
2) Common to Low pass:
a. $6 \mathrm{~dB}$ Cutoff $: 870 \mathrm{MHz}$
b. $\quad 50-870 \mathrm{MHz}: \operatorname{Max} 1.5 \pm 0.5 \mathrm{~dB}$
c. $>1175 \mathrm{MHz}: 15 \mathrm{~dB}$ Min
d. $>1675 \mathrm{MHz}: 45 \mathrm{~dB}$ Min
e. $>1700 \mathrm{MHz}:>20 \mathrm{~dB}$

3) Common to High pass:
a. $6 \mathrm{~dB}$ cut off
: $900 \mathrm{MHz}$
b. $975-1675 \mathrm{MHz} \quad: \operatorname{Max} 2.5 \pm 0.5 \mathrm{~dB}$
c. $>350 \mathrm{MHz}: 15 \mathrm{~dB}$ Min
d. $>675 \mathrm{MHz}: 45 \mathrm{~dB}$ Min
e. $>2100 \mathrm{MHz}:>20 \mathrm{~dB}$

III. Simulations

The return loss of input and output ports are better, greater than $14 \mathrm{~dB}$ and the insertion loss achieved is less than $1 \mathrm{~dB}$ in the MoCA and DVB-C pass bands. The rejection is achieved as per the targeted specifications.

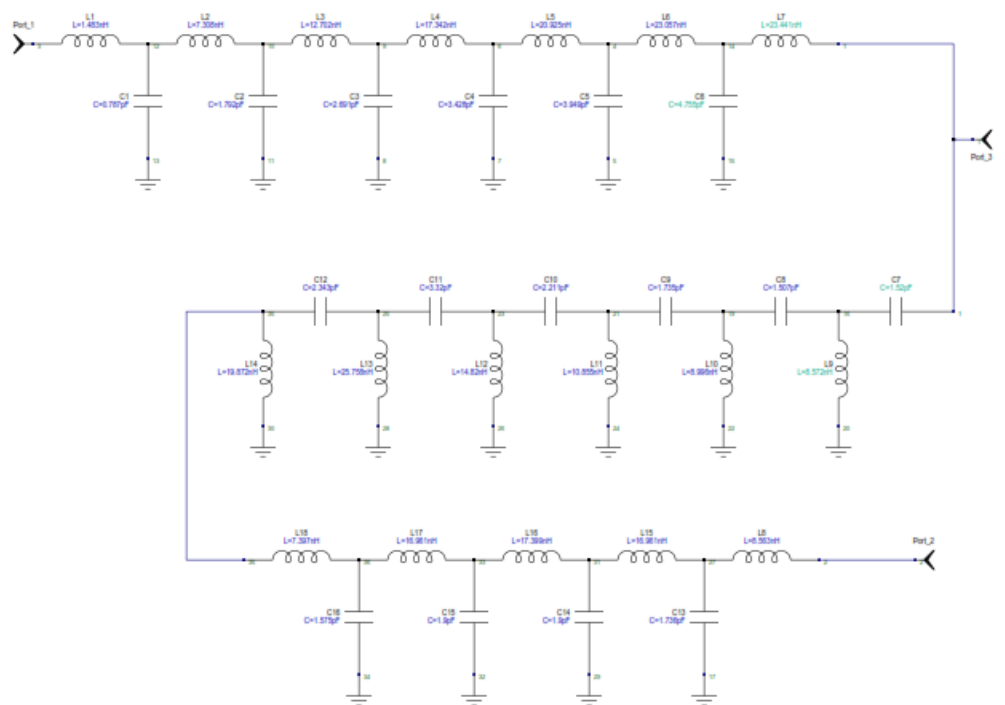

Fig. 3 Simulation circuit diagram

(source: Peter Vizmullar, RF Design Guide -Systems, Circuits and Equations, Artech house, 1995)

From the figure 3, it can be observed that the circuit diagram port 3 is a diplexer input, port 2 is at MoCA side and Port 1 is at DVB-C side.

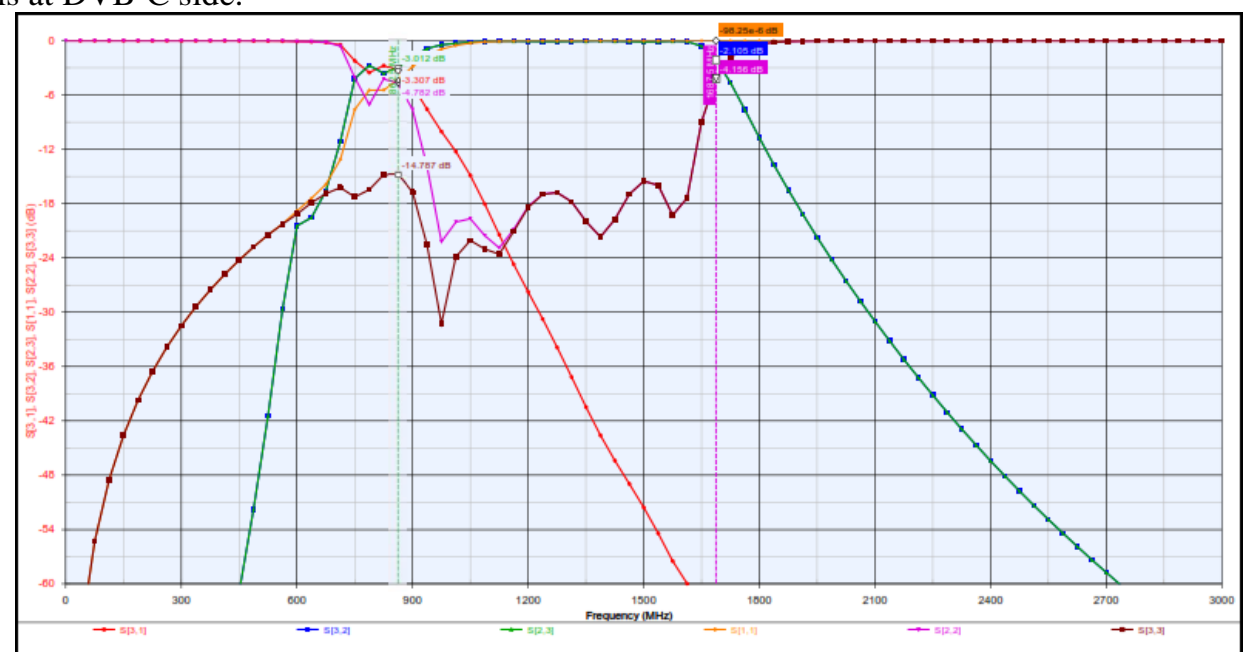

Fig. 4 Result of Simulations 
The following results are observed by the simulated design

- $\mathrm{S}(3,1)$ - Insertion loss of diplexer at Cable port.

- $\mathrm{S}(2,3) \& \mathrm{~S}(3,2)$ - Insertion loss of diplexer at MoCA port

- $\mathrm{S}(1,1)$-Return loss at Cable port

- $\mathrm{S}(2,2)$-Return loss at MoCA port

- $\mathrm{S}(3,3)$ - Return loss at Diplexer Input

\section{Schematics And PCB Layout}

Schematic of diplexer is shown in the figure 5. The modifications are done based on tuning, required to adjust the variations due to tolerances of components and PCB. The DVB-C side of Diplexer consists of 7 section Low pass filter and a Band pass filter on the MoCA side.

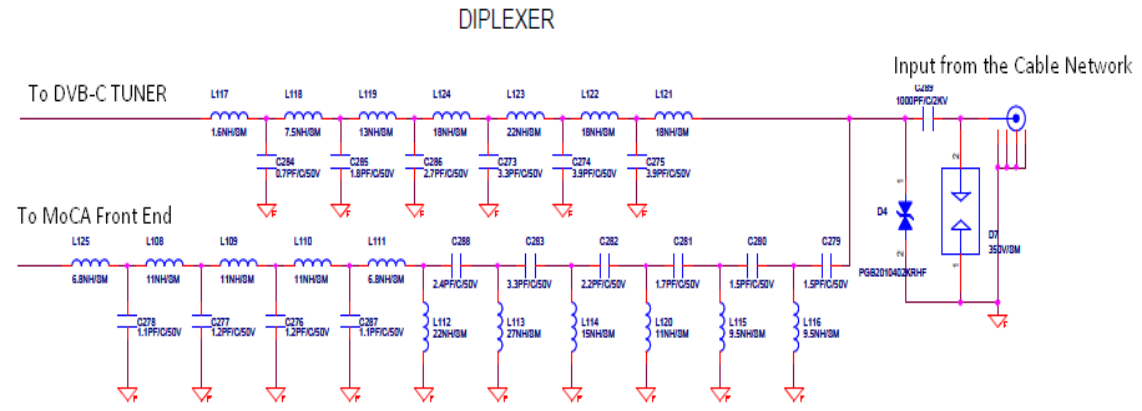

Fig. 5 Schematic of Diplexer (source: Madhusudhan Rao Kothari, Tata Power SED)

The PCB Layout, the Diplexer realized on 4-layer FR4 Board Gerber view is shown in the figure 6.The planned layout is based on the available space on the PCB, rules and previous experience of RF PCB design. The GDT and ESD diodes are placed nearby so that the high voltage storage and Electrostatic charge can be grounded.

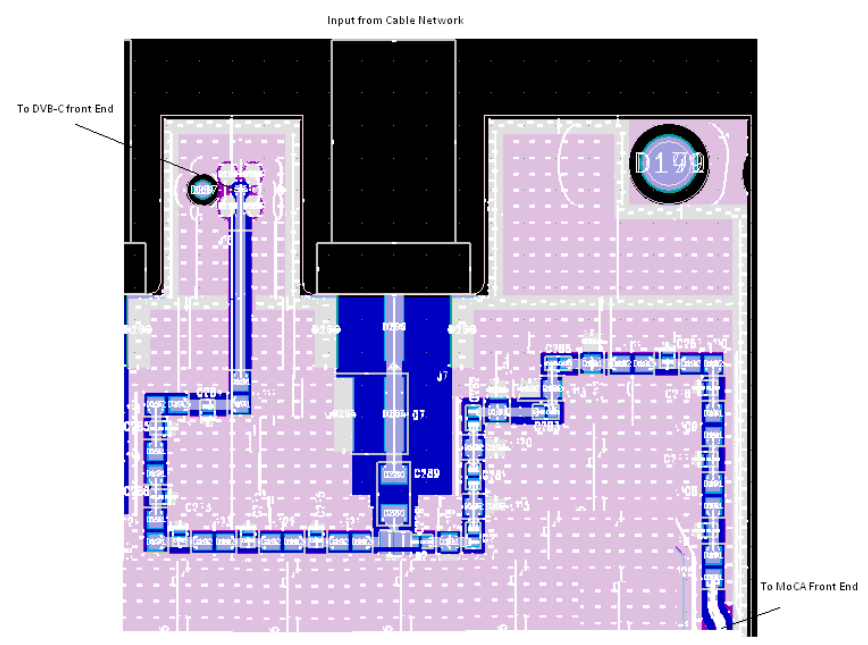

Fig. 6 PCB Gerber View of Diplexer (source: Madhusudhan Rao Kothari, Tata Power SED)

\section{Results And Discussion}

The Figure 7 shows the measured return loss of $9.5 \mathrm{~dB}$ against the required specification of $\geq 8 \mathrm{~dB}$ for a DVB-C/C2 frequency range up to $870 \mathrm{MHz}$ and the required $6 \mathrm{~dB}$ cutoff point is observed to be at $870 \mathrm{MHz}$. 


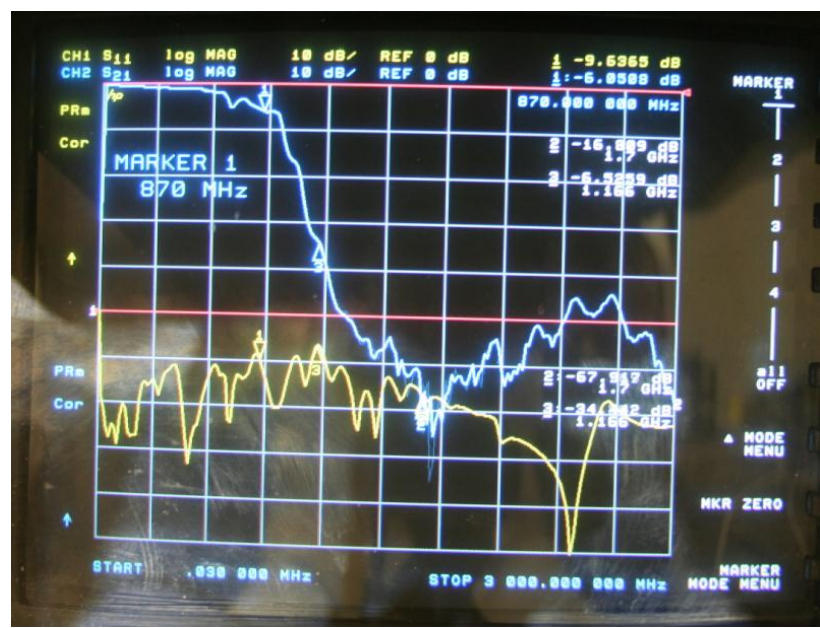

Fig. 7 Measurement showing Return loss at the input of Diplexer and insertion loss at DVB-C/C2 port

The python script used to measure the sensitivity of MoCA is as given in the figure 8. The Figure 9 shows the Measured return loss of $5.4 \mathrm{~dB}$ against the specification of $\geq 5 \mathrm{~dB}$ for required MoCA operating frequency range between 900 to $1675 \mathrm{MHz}$ and the required maximum insertion loss is observed to be in the required frequency range, i.e., is $2.4 \pm 0.5 \mathrm{~dB}$.

From the serial port - set to $1152008 \mathrm{~N} 1$ :
Login: root password: 111111
cd /home/appl/python/techxhome/driver
/MoCADriver.py sync LAN eth1 1 (note: use eth11 for A side MoCA, eth12 for B side on RF card. This will
power the board stack and start the MoCA chiptrying to sync)
/MoCADriver.py filter WAN|LAN2|LAN3|LAN4|LAN5 (note: use WAN for filter 1, LAN2-5 for filter 2-5.
Number after LAN is filter number. Command can be used repeatedly)
/MoCADriver.py disable (this will power off the module stack, should be done before trying any sync command
again)
we can read back the i2c expander switch selection with:
i2cget -y 4390 or you can manually set the GPIOs with:
i2cset -y $4391 \mathrm{X}$ (where $\mathrm{X}$ is the gpio output register value to set in decimal)

Fig. 8 Python script used to measure the sensitivity of MoCA

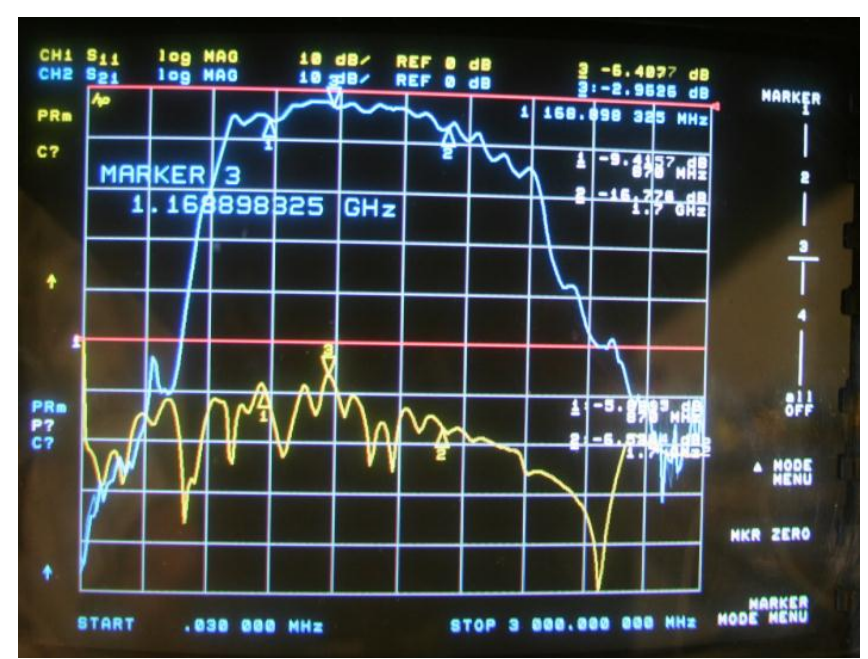

Fig. 9 Measurement showing Return loss at the input of Diplexer and insertion loss at MoCA port 
The measured sensitivity graph is shown in the figure 10 . The physical data rates are measured up to $400 \mathrm{Mbps}$ for a received signal levels of $-52 \mathrm{dBm}$.

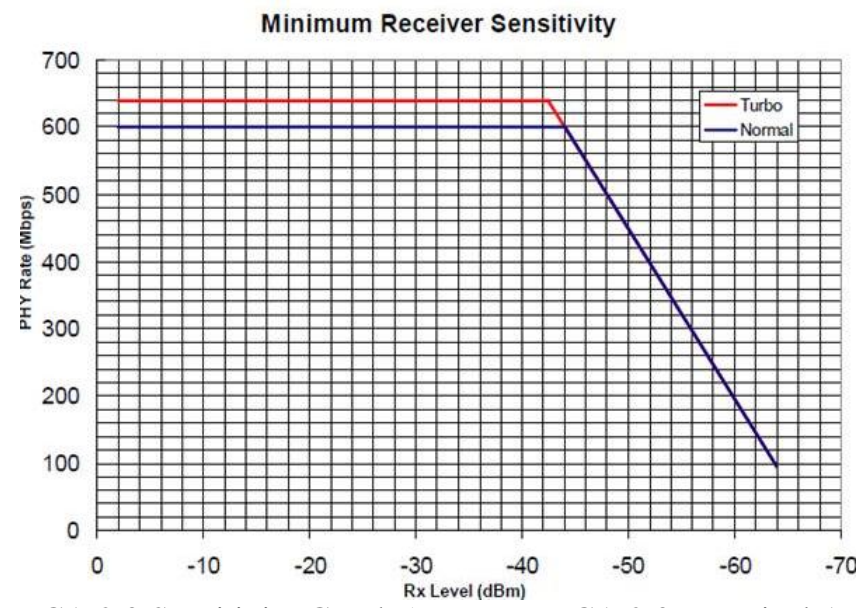

Figure 9.MoCA 2.0 Sensitivity Graph (source: MoCA 2.0, Practical Applications)

The calibrated Automatic Gain Control data after locking 135 channels of DVB-C chip through the diplexer using python script is as follows.

- $\quad$ HNT:/home/appl/python/techxhome/driver\# calibrate NXP 0 -10

- $\quad$ Storing channel 2 frequency 57000000 with adjustment 6.812500

- $\quad$ Storing channel 3 frequency 63000000 with adjustment -6.710000

- $\quad$ Storing channel 4 frequency 69000000 with adjustment -6.440000

- Storing channel 5 frequency 75000000 with adjustment -6.532500

- Storing channel 5 frequency 81000000 with adjustment -6.462500

- Storing channel 5 frequency 87000000 with adjustment -6.445000

- $\quad$ Storing channel 95 frequency 93000000 with adjustment -6.260000

- $\quad$ Storing channel 96 frequency 99000000 with adjustment -6.162500

- Storing channel 97 frequency 105000000 with adjustment -6.102500

- $\quad$ Storing channel 98 frequency 111000000 with adjustment -6.092500

- Storing channel 99 frequency 117000000 with adjustment -5.882500

- Storing channel 14 frequency 123000000 with adjustment -5.725000

- Storing channel 15 frequency 129000000 with adjustment -5.972500

- $\quad$ Storing channel 16 frequency 135000000 with adjustment -5.692500

- $\quad$ Storing channel 17 frequency 141000000 with adjustment -5.682500

- $\quad$ Storing channel 18 frequency 147000000 with adjustment -5.692500

- Storing channel 19 frequency 153000000 with adjustment -5.732500

- Storing channel 20 frequency 159000000 with adjustment -5.552500

- Storing channel 21 frequency 165000000 with adjustment -5.632500

- Storing channel 22 frequency 171000000 with adjustment -5.740000

- $\quad$ Storing channel 7 frequency 177000000 with adjustment -5.890000

- $\quad$ Storing channel 8 frequency 183000000 with adjustment -6.162500

- Storing channel 9 frequency 189000000 with adjustment -6.160000

- Storing channel 10 frequency 195000000 with adjustment -6.432500

- Storing channel 11 frequency 201000000 with adjustment -6.430000

- Storing channel 12 frequency 207000000 with adjustment -6.440000

- $\quad$ Storing channel 13 frequency 213000000 with adjustment -6.485000

- $\quad$ Storing channel 23 frequency 219000000 with adjustment -6.470000

- $\quad$ Storing channel 24 frequency 225000000 with adjustment -6.255000

- Storing channel 25 frequency 231000000 with adjustment -6.025000

- $\quad$ Storing channel 26 frequency 237000000 with adjustment -5.785000

- Storing channel 27 frequency 243000000 with adjustment -5.542500

- $\quad$ Storing channel 28 frequency 249000000 with adjustment -5.812500 
- $\quad$ Storing channel 29 frequency 255000000 with adjustment -5.535000

- $\quad$ Storing channel 30 frequency 261000000 with adjustment -7.752500

- $\quad$ Storing channel 31 frequency 267000000 with adjustment -5.312500

- Storing channel 32 frequency 273000000 with adjustment -5.115000

- Storing channel 33 frequency 279000000 with adjustment -5.025000

- $\quad$ Storing channel 34 frequency 285000000 with adjustment -5.037500

- $\quad$ Storing channel 35 frequency 291000000 with adjustment -5.075000

- $\quad$ Storing channel 36 frequency 297000000 with adjustment -7.822500

- $\quad$ Storing channel 37 frequency 303000000 with adjustment -4.925000

- $\quad$ Storing channel 38 frequency 309000000 with adjustment -4.682500

- Storing channel 39 frequency 315000000 with adjustment -4.525000

- $\quad$ Storing channel 40 frequency 321000000 with adjustment -4.645000

- $\quad$ Storing channel 41 frequency 327000000 with adjustment -4.515000

- Storing channel 42 frequency 333000000 with adjustment -5.135000

- $\quad$ Storing channel 43 frequency 339000000 with adjustment -7.282500

- $\quad$ Storing channel 44 frequency 345000000 with adjustment -7.365000

- $\quad$ Storing channel 45 frequency 351000000 with adjustment -7.422500

- Storing channel 46 frequency 357000000 with adjustment -7.245000

- $\quad$ Storing channel 47 frequency 363000000 with adjustment -7.042500

- $\quad$ Storing channel 48 frequency 369000000 with adjustment -7.580000

- $\quad$ Storing channel 49 frequency 375000000 with adjustment -4.890000

- $\quad$ Storing channel 50 frequency 381000000 with adjustment -7.702500

- $\quad$ Storing channel 51 frequency 387000000 with adjustment -5.252500

- $\quad$ Storing channel 52 frequency 393000000 with adjustment -5.562500

- Storing channel 53 frequency 399000000 with adjustment -5.812500

- Storing channel 54 frequency 405000000 with adjustment -5.862500

- $\quad$ Storing channel 55 frequency 411000000 with adjustment -5.922500

- $\quad$ Storing channel 56 frequency 417000000 with adjustment -5.995000

- $\quad$ Storing channel 57 frequency 423000000 with adjustment -5.545000

- $\quad$ Storing channel 58 frequency 429000000 with adjustment -5.317500

- $\quad$ Storing channel 59 frequency 435000000 with adjustment -5.057500

- Storing channel 60 frequency 441000000 with adjustment -7.630000

- Storing channel 61 frequency 447000000 with adjustment -5.117500

- $\quad$ Storing channel 62 frequency 453000000 with adjustment -7.070000

- Storing channel 63 frequency 459000000 with adjustment -4.120000

- $\quad$ Storing channel 64 frequency 465000000 with adjustment -3.797500

- $\quad$ Storing channel 65 frequency 471000000 with adjustment -3.577500

- Storing channel 66 frequency 477000000 with adjustment -6.717500

- Storing channel 67 frequency 483000000 with adjustment -6.757500

- $\quad$ Storing channel 68 frequency 489000000 with adjustment -4.245000

- $\quad$ Storing channel 69 frequency 495000000 with adjustment -7.095000

- $\quad$ Storing channel 70 frequency 501000000 with adjustment -4.342500

- Storing channel 71 frequency 507000000 with adjustment -7.170000

- $\quad$ Storing channel 72 frequency 513000000 with adjustment -4.202500

- $\quad$ Storing channel 73 frequency 519000000 with adjustment -7.290000

- Storing channel 74 frequency 525000000 with adjustment -7.100000

- Storing channel 75 frequency 531000000 with adjustment -4.370000

- $\quad$ Storing channel 76 frequency 537000000 with adjustment -6.840000

- $\quad$ Storing channel 77 frequency 543000000 with adjustment -3.840000

- Storing channel 78 frequency 549000000 with adjustment -6.930000

- $\quad$ Storing channel 79 frequency 555000000 with adjustment -6.717500

- Storing channel 80 frequency 561000000 with adjustment -7.007500

- $\quad$ Storing channel 81 frequency 567000000 with adjustment -3.955000

- $\quad$ Storing channel 82 frequency 573000000 with adjustment -4.530000

- $\quad$ Storing channel 83 frequency 579000000 with adjustment -4.317500

- $\quad$ Storing channel 84 frequency 585000000 with adjustment -4.617500 
- $\quad$ Storing channel 85 frequency 591000000 with adjustment -4.670000

- $\quad$ Storing channel 86 frequency 597000000 with adjustment -4.732500

- $\quad$ Storing channel 87 frequency 603000000 with adjustment -5.140000

- Storing channel 88 frequency 609000000 with adjustment -4.910000

- $\quad$ Storing channel 89 frequency 615000000 with adjustment -5.420000

- $\quad$ Storing channel 90 frequency 621000000 with adjustment -4.190000

- $\quad$ Storing channel 91 frequency 627000000 with adjustment -6.710000

- Storing channel 92 frequency 633000000 with adjustment -6.480000

- $\quad$ Storing channel 93 frequency 639000000 with adjustment -3.050000

- Storing channel 94 frequency 645000000 with adjustment -3.990000

- Storing channel 100 frequency 651000000 with adjustment -5.270000

- Storing channel 101 frequency 657000000 with adjustment -4.622500

- Storing channel 102 frequency 663000000 with adjustment -4.130000

- Storing channel 103 frequency 669000000 with adjustment -3.920000

- Storing channel 104 frequency 675000000 with adjustment -3.210000

- Storing channel 105 frequency 681000000 with adjustment -6.672500

- Storing channel 106 frequency 687000000 with adjustment -4.650000

- Storing channel 107 frequency 693000000 with adjustment -4.122500

- Storing channel 108 frequency 699000000 with adjustment -5.122500

- Storing channel 109 frequency 705000000 with adjustment -7.135000

- Storing channel 110 frequency 711000000 with adjustment -7.670000

- Storing channel 111 frequency 717000000 with adjustment -6.492500

- Storing channel 112 frequency 723000000 with adjustment -4.672500

- Storing channel 113 frequency 729000000 with adjustment -5.222500

- Storing channel 114 frequency 735000000 with adjustment -4.512500

- Storing channel 115 frequency 741000000 with adjustment -4.802500

- Storing channel 116 frequency 747000000 with adjustment -4.592500

- Storing channel 117 frequency 753000000 with adjustment -4.610000

- Storing channel 118 frequency 759000000 with adjustment -4.390000

- Storing channel 119 frequency 765000000 with adjustment -4.160000

- Storing channel 120 frequency 771000000 with adjustment -4.920000

- Storing channel 121 frequency 777000000 with adjustment -3.927500

- Storing channel 122 frequency 783000000 with adjustment -3.460000

- Storing channel 123 frequency 789000000 with adjustment -3.247500

- Storing channel 124 frequency 795000000 with adjustment -2.917500

- Storing channel 125 frequency 801000000 with adjustment -2.320000

- $\quad$ Storing channel 126 frequency 807000000 with adjustment -2.147500

- $\quad$ Storing channel 127 frequency 813000000 with adjustment -2.710000

- Storing channel 128 frequency 819000000 with adjustment -2.260000

- $\quad$ Storing channel 129 frequency 825000000 with adjustment -2.310000

- $\quad$ Storing channel 130 frequency 831000000 with adjustment -2.160000

- $\quad$ Storing channel 131 frequency 837000000 with adjustment -1.960000

- $\quad$ Storing channel 132 frequency 843000000 with adjustment -2.287500

- $\quad$ Storing channel 133 frequency 849000000 with adjustment -1.050000

- $\quad$ Storing channel 134 frequency 855000000 with adjustment -0.700000

- Storing channel 135 frequency 861000000 with adjustment -0.152500

\section{Conclusion}

In this study, the design adapted for the diplexer is a universal approach and can be used in other similar applications with high power handling capability. The diplexer design can be realized in a required PCB form factor for further Miniaturization. Also the same design can be tried out using RFMEMS for better results and for reduction in the size. The cost of diplexer can be optimized by selecting the low cost inductors and capacitors. The return loss of input port is limited because of the GDT internal capacitance and F-Connector. For better input return loss, these devices can be replaced with better specifications and for achieving better rejections, the inductors with Higher Q-factors are suggested. 


\section{Acknowledgements}

The authors would like to thank Mr. Chalapathi Subramanyam for supporting with the required Python test scripts for MoCA and DVB-C/C2 chips. These are used in the integration testing of the Diplexer with DVB-C and MoCA Systems. Also thanks to Dr. V. Venkateswarlu and Dr. Siva Yellampalli for the discussions and encouragement.

\section{References}

[1] J. Sarma, Technical Interoperability of DTH Set top Boxes, Telecom Regulatory Authority of India, 11, 2010, 1-33

[2] 2nd Generation Cable: The World's Most Advanced Digital Cable TV System, DVB Fact Sheet - July 2012

[3] MoCA 1.1 Specification for Device RF Characteristics, 20142011

[4] MoCA home network installation and maintenance, Sprient Application notes, Rev B. 07/10.

[5] MoCA 2.0:Practical Applications, Entoripic Communications, 2011

[6] Ulrich Reimers, Digital Video Broadcasting (DVB) the future of television, Physics World, 1998.

[7] Peter Vizmullar, RF Design Guide -Systems, Circuits and Equations, Artech house, 1995

[8] CCBN cable Digital TV operator Summit, Beijing, Rob Gelphman, March 2014. 